\title{
INFLUENCE OF HALF-SQUAT INTENSITY AND VOLUME ON THE SUBSEQUENT COUNTERMOVEMENT JUMP AND FREQUENCY SPEED OF KICK TEST PERFORMANCE IN TAEKWONDO ATHLETES
}

\author{
Jonatas Ferreira da Silva Santos ${ }^{1}$, Tomás Herrera-Valenzuela ${ }^{2,3}$, \\ Gustavo Ribeiro da $\mathrm{Mota}^{4}$, and Emerson Franchini ${ }^{1}$ \\ ${ }^{1}$ Martial Arts and Combat Sports Research Group, Sport Department, \\ School of Physical Education and Sport, University of São Paulo, Brazil \\ ${ }^{2}$ Faculty of Sciences for Physical Activity, Universidad San Sebastián, Chile \\ ${ }^{3}$ Laboratory of Sciences Physical Activity, Sport and Health, \\ Universidad de Santiago de Chile, USACH, Chile \\ ${ }^{4}$ Human Performance and Sport Research Group, Postgraduate Program in Physical Education, \\ Federal University of Triângulo Mineiro, Uberaba, MG, Brazil
}

Original scientific paper

UDC: 796.856:796.012.1

\begin{abstract}
:
The aim of this study was to assess the effects of different postactivation potentiation conditioning activities' volumes and intensities on countermovement jump and multiple sets of high speed kicks. Nine taekwondo athletes $(\mathrm{M} \pm \mathrm{SD}$; age: $20.3 \pm 5.2$ years; height: $177 \pm 7.2 \mathrm{~cm}$; body mass: $71.8 \pm 15.3 \mathrm{~kg}$; maximum dynamic half-squat 1RM: $132.8 \pm 32.5 \mathrm{~kg}$ and practice time: $9.6 \pm 7.2$ years) participated. One control and four experimental conditions were randomly applied. Each condition was composed of warm-up, conditioning activity (half-squat: $1 \times 3$ at 50 or $90 \% 1 \mathrm{RM}$ or $3 \times 3$ at 50 or $90 \% 1 \mathrm{RM}$ ), followed by a 10 -minute rest interval, a countermovement jump and five sets of Frequency Speed of Kick Test. The conditions were compared using an analysis of variance with repeated measures, followed by Bonferroni post-hoc test. The alpha level was set at $5 \%$. The significant difference was found in the number of kicks among sets $\left(\mathrm{F}_{3,21 ; 128,36}=25.34 ; \mathrm{p}<.001\right.$; $\eta^{2}=.388$ [small]). The rating of perceived exertion before control condition was higher than in all experimental protocols $\left(\mathrm{F}_{4,32}=6.64 ; \mathrm{p}=.001 ; \eta^{2}=.454\right.$ [small]). There were no effects of volume and intensity on the variables investigated (maximum countermovement jump, mean countermovement jump, kick decrement, impact and rating of perceived exertion). Our results indicate that taekwondo athletes does not improve performance after conditioning activities of different volumes and intensities.
\end{abstract}

Key words: postactivation potentiation, resistance exercise, warm-up

\section{Introduction}

Taekwondo athletes use both offensive and defensive techniques, involving punches and kicks (Kazemi, Waalen, Morgan, \& White, 2006). In total, $98 \%$ of applied technical actions originate from the lower limbs (Kazemi, et al., 2006), and approximately $81 \%$ of strikes are intended to reach the opponent's trunk (Kwok, 2012). The main technique used during taekwondo matches is the bandal tchagui (Kazemi, et al., 2006), which consists of a semicircle kick in the air to reach the opponent's trunk with the foot. The bandal tchagui is performed at a high speed and with great power (Pieter $\&$ Pieter, 1995), making it difficult for the opponent to defend or counterattack and resulting in points gained during the match. It has been shown that more experienced athletes performed the bandal tchagui faster than less experienced ones (Ha, Choi, $\& \mathrm{Kim}, 2009$ ). This is probably one of the reasons why medal-winning taekwondo athletes used this technique more ( $72.8 \%$ of total strikes applied) than non-medalists (50.3\% of total strikes applied) in the Asian Games (Kwok, 2012).

For the activities demanding high muscle power, the postactivation potentiation (PAP) has been described as an important strategy to improve performance (Sale, 2004; Tillin \& Bishop, 2009; Wilson, et al., 2013). The PAP refers to an acute 
increase in performance caused by a previous conditioning activity. Recent research has shown that magnitude of intensity, volume, and rest interval applied before the main activity and the subject characteristic can affect PAP (Wilson, et al., 2013). Athletes have a greater chance to manifest PAP than untrained or trained non-athletes (Wilson, et al., 2013). It has been noted that exercise execution with moderate loads (60-84\% 1RM), in multiple sets and with rest interval of between seven and 10 minutes, is more suitable for PAP manifestation than exercise execution with high loads (85-100\% 1RM), in a single set and with less than two minutes or more than 10 minutes rest intervals (Wilson, et al., 2013). However, even following these recommendations, inconsistent results have been observed (Tillin \& Bishop, 2009). Sale (2004) attributes the inconsistency of results from PAP manifestation to the characteristics of conditioning activity and rest interval applied before the main activity, mainly for two reasons: first, a more intense prolonged exercise can induce fatigue and second, a long rest recovery interval applied before the main activity reduces or eliminates fatigue effects and can also dissipate PAP effect. Moreover, it is important to know the physiological response to a given training stimulus and its influence on the performance of the next activity.

A countermovement jump (CMJ) is used to measure muscle power of lower limbs in investigations about PAP in different sports (Gouvêa, Fernandes, César, Silva, \& Gomes, 2013; Wilson, et al., 2013); among taekwondo athletes, CMJ is the primary measure used to evaluate lower limb muscle power (Bridge, Santos, Chaabène, Pieter, \& Franchini, 2014). However, research investigating the effects of PAP on combat sports such as judo (Miarka, Del Vecchio, \& Franchini, 2011) and taekwondo (Santos, Valenzuela, \& Franchini, 2015) used specific gestures made after completion of the conditioning activity and observed improved performance. In a study with taekwondo athletes an increased performance in frequency of speed kick test (FSKT) was reported after a complex exercise followed by 10-minute rest intervals (Santos, et al., 2015). However, no studies have investigated the effect of volume and intensity of prior exercises on the performance in multiple sets of specific and non-specific gestures in taekwondo athletes. Therefore, the purpose of this study was to investigate the volume and intensity effects on performance of $\mathrm{CMJ}$ and on the number and impact force of kicks generated during five sets of FSKT. It was hypothesized that athletes would perform better after a pre-test exercise of a higher volume and intensity and only in the specific activity.

\section{Methods}

\section{Sample}

Nine black-belt taekwondo athletes $(\mathrm{M} \pm \mathrm{SD}$; age: $20.3 \pm 5.2$ years; height: $177 \pm 7.2 \mathrm{~cm}$; body mass: $71.8 \pm 15.3 \mathrm{~kg}$; maximum dynamic half-squat $1 \mathrm{RM}$ : $132.8 \pm 32.5 \mathrm{~kg}$ and practice time: $9.6 \pm 7.2$ years) volunteered to participate in this study and provided written consent after being informed about the procedures and risks associated. The athletes competed within the state or at more prominent levels (international quality athletes: 6 ; national: 1 ; state: 2) and resistance trained one to three times per week (depending on their training periodization phase for a minimum of one year) and participated in taekwondo sessions $5 \pm 1$ times per week, which each lasted for two hours. They were free from any lower body injury or neuromuscular disorder. The research was approved by the Institutional Ethics Committee.

\section{Experimental procedures}

This study used a within-subject repeated measures research design to investigate the effect of conditioning activities' intensity and volume on CMJ and FSKT performance. This study consisted of four experimental protocols and a control proto$\mathrm{col}$. The order of execution of all protocols was randomly determined. Half-squat resistance exercise was used in all experimental protocols. Protocol one was composed of $1 \times 3$ repetitions at $50 \%$ of 1RM (low-volume and low-intensity - LL); protocol two consisted of $1 \times 3$ at $90 \%$ of $1 \mathrm{RM}$ (lowvolume and high-intensity - LH); protocol three consisted of $3 \times 3$ repetitions at $50 \%$ of 1RM (highvolume and low-intensity - HL); and protocol four consisted of $3 \times 3$ repetitions at $90 \%$ of 1RM (highvolume and high-intensity $-\mathrm{HH}$ ). Ten minutes after the completion of any experimental protocol CMJ and FSKT were performed. The same athletes performed all protocols in independent sessions with a minimum interval of 48 hours between each experimental session.

This study lasted three weeks. In the first week, the subjects were familiarized with the procedures used. Familiarization had the following purposes: a) to guide and familiarize the athletes with the correct technique for applying FSKT, CMJ, 1RM during all experimental procedures; $b$ ) to determine the correct body position of athletes in motor actions that were used. The athletes received guidance about the technical execution of CMJ and, further, they were asked to perform a set of jumps (three to five jumps) in order to consolidate learning technique. The athletes were also familiar with the 
procedures involved in maximal dynamic strength testing of the lower limbs. The 1RM test was performed using the half-squat exercise.

In the second week, the athletes performed $1 \mathrm{RM}$ tests using half-squat exercise. In the third week, the athletes performed the experimental procedures. All experimental sessions were initiated by the standardized general warm-up. The general warm-up was composed of five minutes running on the treadmill at speeds between eight and nine $\mathrm{km} / \mathrm{h}$ followed by a two-minute rest interval. After the warm-up, the athletes performed a control and four experimental protocols (strength condition: one or three sets and intensity of 50 or $90 \%$ of 1RM). Before applying CMJ and multiple FSKT a 10 -minute rest interval was given. This rest interval was used because a recent meta-analysis (Wilson, et al., 2013) indicated that rests between seven and 10 minutes are suitable to find PAP manifestation. The study was conducted on five different days, resulting in only one experimental protocol realized per day. A rest interval of 48 hours was given between each experimental protocol execution.

\section{Warm-up and exercise protocols}

Control protocol. After the warm-up the athletes waited for two minutes and then performed a CMJ and multiple-set FSKT.

Strength protocols (half-squat resistance exercises). The half-squat resistance exercise was performed in the following ways: $1 \times 3$ at 50 or $90 \%$ $1 \mathrm{RM}$ and $3 \times 3$ at 50 or $90 \% 1 \mathrm{RM}$, followed by a 10 -minute rest interval, and then a CMJ and five consecutive FSKT tests were performed.

\section{Performance assessments}

One-repetition maximum test. The maximal dynamic strength for lower body was measured by a half-squat resistance exercise. All the subjects performed a familiarization session prior to the 1RM session using Olympic barbells $(20 \mathrm{~kg})$ and free weights. The half-squat movement was performed according to standard procedures. General warm-up consisted of five minutes running on a horizontal treadmill at a constant speed of nine $\mathrm{km} / \mathrm{h}$ followed by a three-minute rest interval. After, the subjects performed two sets of the half-squat resistance exercise. The first set consisted of eight repetitions with $50 \%$ of the estimated 1RM followed by the second set of three repetitions at $70 \%$ of the estimated 1RM with a three-minute rest interval between sets. The 1RM load was determined after a maximum of five attempts (Brown \& Weir, 2001).

Counter movement jump. The CMJ was performed on a contact platform (Axon Jump ${ }^{\circledR}$, Axon Sports Bioengineering, Argentina). Each subject performed three CMJs. Both the maximal jump height and the mean jump height were considered for later analysis. To exclude the influence of arm swing, the subjects were instructed to keep their hands on their waist and encouraged to jump as high as possible. A short rest ( $\sim 15$ seconds) was given after each CMJ until completing the third jump. Special attention of evaluators was focused on the correct jump performance, that is, no flexion or extension of the lower limbs was allowed. The CMJ test was reliable enough (intraclass correlation coefficient $[\mathrm{ICC}]=0.97$ ) and the coefficient of variation was $3.4 \%$. The ICC and coefficient of variation were calculated within session between multiple CMJs.

Multiple frequency speed of kick test. Each FSKT lasted ten seconds. During this study, each athlete performed five FSKT with a 10-second rest interval between each repetition after every pre-test protocol. To perform FSKT, each athlete assumed position in front of the stand bag equipped with a taekwondo trunk protector (TK-Strike Protector ${ }^{\circledR}$, Daedo, Barcelona, Spain) (Figure 1a). After the audio signal, the subject performed the maximum number of kicks, alternating the right and left leg (Figure 1b). The body protector was activated when the sensors positioned in athletes' socks touched the sensors in the body protector. For each weight category and gender there is a specific minimum impact value that should be achieved for the technique to be validated as a score (Del Vecchio, Franchini, Del Vecchio, \& Pieter, 2011). The technique used during the test was a turning kick (bandal tchagui). The performance on test was determined by the total number of kicks and the maximal impact generated during the test. Multiple-set FSKT presented $\mathrm{M} \mathrm{ICC}$ of .85 between the test and retest. The mean coefficient of variation between test and retest was $3.9 \%$. The ICC and coefficient of variation were calculated based on previous unpublished data from our laboratory.

Rating of perceived recovery. This approach has been utilized to assess athlete's recovery level.. The rating of perceived recovery (RPR) scale, proposed by Laurent et al. (2011), was applied prior to either experimental or control protocol. The main purpose of this particular scale is to detect early signs of overtraining (Laurent, et al., 2011). However, in our study, it was used to detect the athletes' perception of their recovery before the implementation of different experimental protocols. The RPR scale is similar (from zero - very poorly recovered/extremely tired to 10 - very recovered/highly energetic) to RPE scale, representing varying levels of an individual's level of RPR.

Session-rating of perceived exertion. The session-RPE scale (CR-10), proposed by Foster et al. (2001), was applied 30 minutes after each experimental and control protocols. The instructions on the use of perceived exertion scale was read to the participants prior to the test. The subjects were 
A

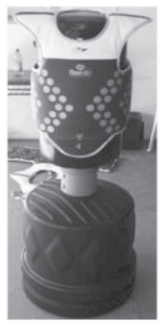

B
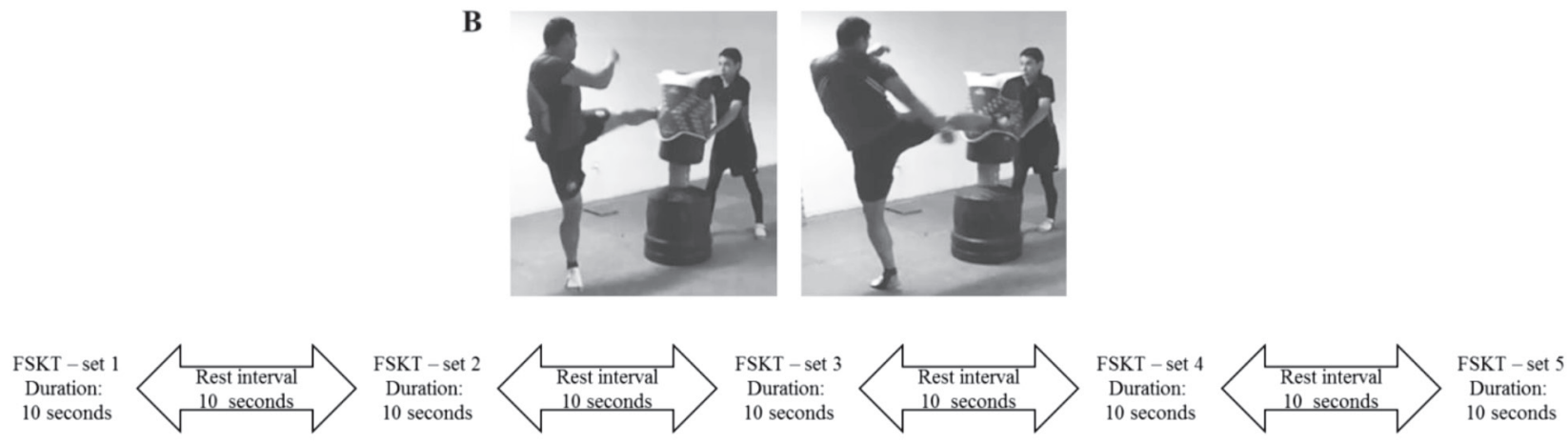

Figure 1. Stand bag with a taekwondo trunk protector (A) and multiple-set frequency speed of kick test (FSKT) execution (B).

asked to rate how much they perceived their exertion. The session-RPE is a zero (rest) to 10 (maximal exertion) scale.

Kick decrement. The kick decrement indicates performance decrease during the FSKT. To calculate the kick decrement the number of blows applied during the multiple-set FSKT was taken into account. The equation takes into account the results of all FSKT sets (Equation) (Girard, Mendez-Villanueva, \& Bishop, 2011).
$.1-<.3$ [small]; .3-<.5 [moderate]; .5-<.7 [large]; .7-< .9 [very large]; .9-1 [perfect] (Hopkins, 2013). For all analyses we adopted $\alpha=5 \%$. All analyses were conducted using Statistica 10 for Windows.

\section{Results}

Generally, for the five sets of FSKT there was no effect of the experimental protocols $\left(\mathrm{F}_{4.40}=.954\right.$; $\mathrm{p}=.443, \eta^{2}=.087$ [trivial]) or no interaction between

$$
\text { Kick decrement }(\%)=\frac{[1-(\text { FSKT1 }+ \text { FSKT2 + FSKT } 3+\text { FSKT4 + FSKT 5) }] \times 100}{\text { Best FSKT set } x \text { number of sets }}
$$

(Equation)

The athlete who performed more blows got an index of performance more consistent with his/her performance.

\section{Statistical analysis}

All results are expressed as $\mathrm{M} \pm \mathrm{SD}$. After analysis of the sphericity of the data via Mauchly's test, one-way analysis of variance (ANOVA) with repeated measures was performed to compare the dependent variables (CMJ, FSKT, kick decrement, RPR and RPE) across different interventions. Greenhouse-Geisser test was used when the assumption of sphericity of the data was not confirmed. Pre-test exercise protocols and multiple-set FSKT were compared using a two-way ANOVA with repeated measures. When differences were detected, the Bonferroni test was used as a post$h o c$ analysis. The effect size values were also calculated using eta squared $\left(\eta^{2}\right)$ and classified using the following scale for interpretation: $<.1$ [trivial];

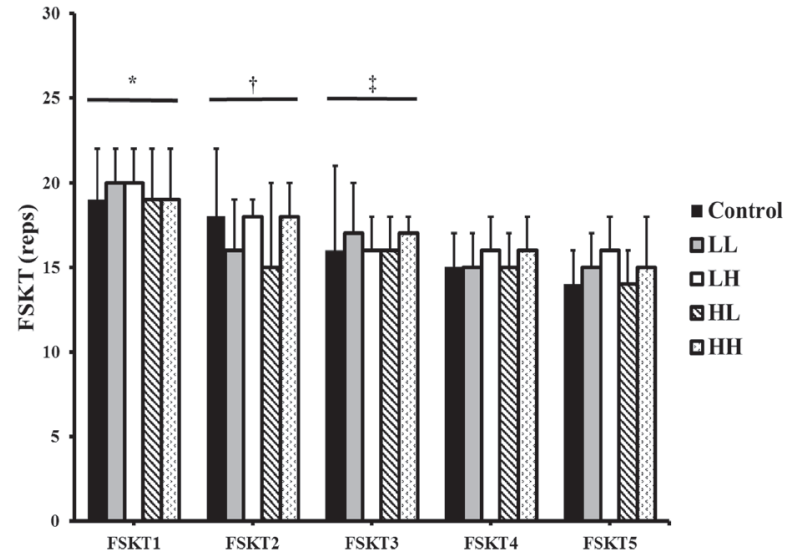

LL: low-volume and low-intensity; LH: low-volume and highintensity; HL: high-volume and low-intensity; $\mathrm{HH}$ : high-volume and high-intensity; *: different from the other conditions; $\dagger$ : different from FSKT4 $(p=.039)$ and FSKT5 $(p=.002)$; $\ddagger$ : different from FSKT5 $(p=.010)$.

Figure 2. Frequency Speed of Kick Test (FSKT) repetitions $(n=9)$. Data are presented as $M \pm S D$. 

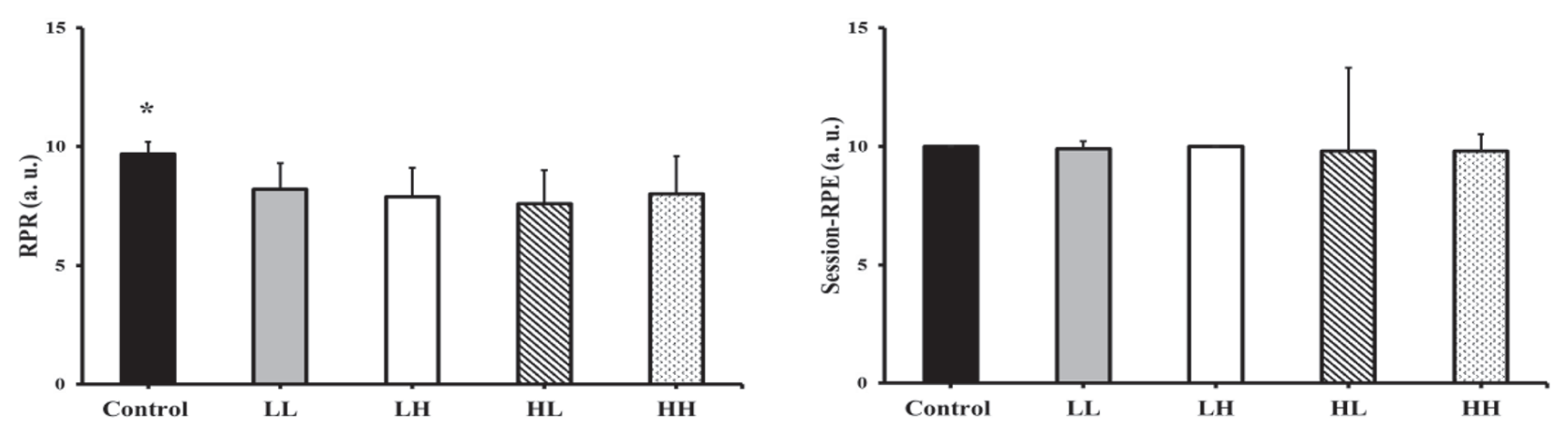

LL: low-volume and low-intensity; LH: low-volume and high-intensity; HL: high-volume and low-intensity; HH: high-volume and highintensity; RPR = rating of perceived recovery; Session-RPE = rating of perceived exertion in a training session; a.u.: arbitrary unit; *: different from LL, LH, HL and $\mathrm{HH}$ at the same moment $(p<.05)$.

Figure 3. Rating of perceived recovery (RPR, Panel A) and rating of perceived exertion ( $R P E$, Panel $B)(n=9)$. Data are presented as $M \pm S D$.
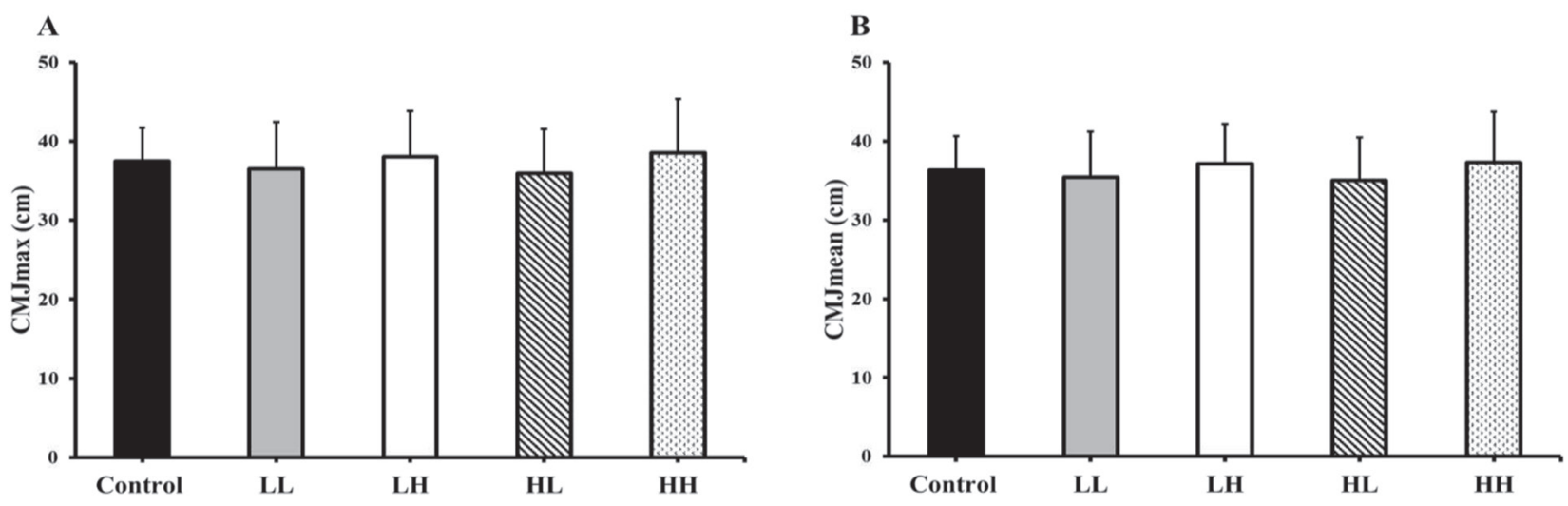

LL: low-volume and low-intensity; LH: low-volume and high-intensity; HL: high-volume and low-intensity; HH: high-volume and highintensity.

Figure 4. Maximum (A) and mean (B) countermovement jump performances after different experimental procedures $(n=9)$. Data are presented as $M \pm S D$.

protocol and multiple-set FSKT $\left(\mathrm{F}_{16,160}=.558 ; \mathrm{p}=.911\right.$, $\eta^{2}=.053$ [trivial]), but there was an effect of the sets $\left(\mathrm{F}_{3,21}, 128,36=25.344 ; \mathrm{p}<.001, \eta^{2}=.388\right.$ [moderate] $)$, with higher values in the first set compared to the second $(p=.002)$, third, fourth and fifth sets $(\mathrm{p}<.001$ for all comparisons), higher in the second set than in the fourth $(\mathrm{p}=.036)$ and fifth sets $(\mathrm{p}=.001)$ and higher in the third set compared to the fifth $(p=.010)$ (Figure 2).

There was an effect of the protocols on RPR $\left(\mathrm{F}_{4,32}=6.64 ; \mathrm{p}=.001, \eta^{2}=.454\right.$ [moderate] $)$, with higher values in the control protocol compared to LL $(p=.030)$, LH $(p=.004)$, LH $(p<.001)$ and HH $(p=.008)$ protocols. However, there was no effect of protocol on session-RPE $\left(\mathrm{F}_{4,32}=.899 ; \mathrm{p}=.476, \eta^{2}=.101\right.$ [small]) (Figure 3).

An effect of protocols was observed on the maximum height achieved in $\mathrm{CMJ}\left(\mathrm{F}_{4,32}=2.71\right.$; $\mathrm{p}=.047, \eta^{2}=.253$ [small]); however, the Bonferroni test did not identify any difference between experimental protocols and the effect size was small. The same result was observed for the average height attained in three jumps $\left(\mathrm{F}_{4,32}=3.11 ; \mathrm{p}=.0286, \eta^{2}=.28\right.$ [small]), but again the Bonferroni test did not identify any difference between experimental protocols and the effect size was small (Figure 4).

No effect of protocols on the total number of kicks (control: 82 \pm ; LL: $83 \pm 7$; LH: $85 \pm 4$; HL: 79 \pm 9 ; HH: $85 \pm 8)\left(\mathrm{F}_{4,32}=1.00 ; \mathrm{p}=.418, \eta^{2}=.112\right.$ [small]), kick decrement (control: $19.2 \pm 7.9 \%$; LL: $16.8 \pm 7.7 \%$; LH: $15.7 \pm 6.0 \%$; HL: $16.5 \pm 7.3 \%$; HH: $13.8 \pm 5.7 \%$ ) $\left(\mathrm{F}_{4,32}=.79 ; \mathrm{p}=.541, \eta^{2}=.09\right.$ [trivial] $)$ and impact force of the kicks during multiple FSKT (control: $59.8 \pm 7.0$ a.u.; LL: $59.8 \pm 4.8$ a.u.; LH: $60.3 \pm 5.3$ a.u.; HL: $60.6 \pm 3.5$ a.u.; $H H$ : $62.4 \pm 4.3$ a.u. $)\left(\mathrm{F}_{4,32}=.541\right.$; $\mathrm{p}=.707, \eta^{2}=.063$ [trivial]) was observed.

\section{Discussion and conclusions}

The aim of this study was to investigate the effects of different postactivation potentiation conditioning activity's volumes and intensities on CMJ and multiple sets of FSKT. We hypothesized that a more intense and higher volume resistance exer- 
cise would be more effective in improving performance of taekwondo athletes. However, this hypothesis was not confirmed. The primary finding of this study was that neither volume nor intensity affected performance on taekwondo-specific and non-specific tests. There was a consistent reduction in the number of kicks across sets of FSKT regardless of the experimental protocol used. In the first sets a higher number of kicks were performed than in the fourth and fifth sets, and there were more kicks applied in the third sets than in the fifth ones. However, the effect size presented was small. The athletes reported being more recovered before the control protocol than before other experimental protocols used - the effect size was moderate. There was an effect of the experimental protocols on the average and maximum CMJ height; however, this effect size was small. The variables (the total number of kicks, kick decrement, impact force, and $\mathrm{RPE}$ ) were not affected by the volume (number of sets) or intensity of pre-test resistance exercise used as the conditioning activity.

The effect of different conditioning actions on FSKT performance was investigated in a previous study (Santos, et al., 2015). However, our study is the first one that investigated the effect of volume (number of sets) and intensity of a resistance exercise on performance in taekwondo-specific and non-specific tests. Recently, it has been noted that for the PAP manifestation, activities composed of multiple sets are superior to single-set activities and that moderate loads (60-84\% 1RM) are superior to high loads ( $85-100 \%$ 1RM) (Wilson, et al., 2013). Another possible reason for the lack of improvement in performance after the application of different protocols used in our study is related to the resistance training experience of the athletes. Athletes with a more than three-year experience in strength training are more responsive to conditioning activities (Wilson, et al., 2013) because the effects of fatigue are likely to be lower in highly trained athletes than in practitioners (Kilduff, et al., 2007; Wilson, et al., 2013). As the taekwondo athletes in our study had a minimum of one year of strength training experience, perhaps they could not be considered highly strength-trained.

Another acute variable manipulated in the PAP research is duration of rest interval (Tillin \& Bishop, 2009; Wilson, et al., 2013; Santos, et al., 2015). The rest interval used in our study (10 minutes) has also been used in several studies investigating the expression of PAP, and an improvement in performance has been reported (Tillin \& Bishop, 2009; Wilson, et al., 2013), including a taekwondo PAP study (Santos, et al., 2015). Furthermore, it has been suggested that rest intervals between three and 12 minutes are more suitable when the goal is the manifestation of PAP (Gouvêa, et al., 2013; Wilson, et al., 2013). Sale (2004) attributes the inconsistency of results from the manifestation of the characteristics of the PAP activity and the interval applied before the main activity to two reasons: 1) prolonged intensive exercise not only activates the mechanisms of PAP but also produces great amount of fatigue; 2) long-lasting recovery times applied before the main activity would reduce or eliminate the effects of fatigue, but it may also dissipate the effects of PAP.

In repeated short exercise, it has been reported that fatigue develops rapidly after the first set (Mendez-Villanueva, Hamer, \& Bishop, 2008). Our study showed a decrease in performance over the five sets of FSKT. Performance was higher in the first set than in the others, higher in the second set than in the fourth and fifth and higher in the third set than in the fifth. No other study used the repeated FSKT five times, thus, we cannot compare our results with those of other investigations. However, while the experimental protocols used in this study did not change performance during the main activity (FSKT), there was also no increase in fatigue. This result suggests that strength training did not negatively affect performance in repeated short exercise using frequency of kicks as activity and can be used before a specific taekwondo training. The study conducted by Miarka et al. (2011) reported improved performance in the first period of the Special Judo Fitness Test (a repeated short test) after an acute strength training.

In taekwondo, the main purpose is to knock out the opponent or score points, therefore the kick impact force is very important. To score in taekwondo, athletes need to reach the opponent with at least a minimum impact force specific to their weight category. In our study, there was no statistical difference in the impact force generated during FSKT between the different experimental protocols. Similar results were described in the literature using FSKT (Santos, et al., 2015). Other studies investigated different distances and time to improve the impact force generated during the execution of the roundhouse kick to the head (Falco, et al., 2009; Estevan, Álvarez, Falco, Molina-García, \& Castilho, 2011; Estevan, Falco, Álvarez, \& MolinaGarcía, 2012). These studies noted that weight category (featherweight vs. heavyweight), experience time (medalists vs. non-medalists; experts vs. novices), and distances of execution (short, medium, long) can affect the impact force between taekwondo athletes (Falco, et al., 2009; Estevan, et al., 2011, 2012). However, only one study investigated the PAP manifestation on impact generated during taekwondo kicks (Santos, et al., 2015).

The athletes had a higher RPR before the control protocol testing compared to those before all experimental protocols. Because the execution order of protocols was randomized and the athletes did not know which activity would be conducted on a specific testing day, it is not possible to explain 
this result. Although there have been significant differences between values of the reported RPR, they were all in the range where no decrease in performance is expected (Laurent, et al., 2011). Indeed, in our study, all values indicated by the athletes on the RPR scale were higher than seven; thus, an increased performance was expected. There was no statistical difference in session-RPE after the completion of the experimental protocols. This indicates that the athletes perceived that all the experimental protocols resulted in similar exertion.

The findings of this study indicate that performance in FSKT was not altered by either volume or intensity of the pre-test conditioning activity across sets when a 10-minute rest was used. In repeated short exercise, like of FSKT, it has been reported that fatigue rapidly develops after the first set. Performing strength exercises with various intensities and volumes did not affect performance in the main activity (FSKT). However, the use of strength exercises did not decrease strength of the subsequent performance. Thus, coaches can use exercises with the same characteristics as those used in this study, providing combined stimuli to the athlete without any change in performance during the intermittent exercise.

\section{References}

Bridge, C.A., Santos, J.F.S., Chaabène, H., Pieter, W., \& Franchini, E. (2014). Physical and physiological profiles of taekwondo athletes. Sports Medicine, 44(6), 713-733.

Brown, L.E., \& Weir, J.P. (2001). ASEP Procedures recommendation I: Accurate assessment of muscular strength and power. Journal of Exercise Physiology, 4(3), 1-21.

Del Vecchio, F.B., Franchini, E., Del Vecchio, A.H.M., \& Pieter, W. (2011). Energy absorbed by electronic body protectors from kicks in a taekwondo competition. Biology of Sport, 28(1), 75-78.

Estevan, I., Álvarez, O., Falco, C., Molina-Garcia, J., \& Castilho, I. (2011). Impact force and time analysis influenced by execution distance in a roundhouse kick to the head in taekwondo. Journal of Strength and Conditioning Research, 25(10), 2851-2856.

Estevan, I., Falco, C., Alvarez, O., \& Molina-García, J. (2012). Effect of Olympic weight category on performance in the roundhouse kick to the head in taekwondo. Journal of Human Kinetics, 31, 37-43.

Falco, C., Alvarez, O., Castillo, I., Estevan, I., Martos, J., Mugarra, F., et al. (2009). Influence of the distance in a roundhouse kick's execution time and impact force in taekwondo. Journal of Biomechanics, 42(3), 242-248.

Foster, C., Florhaug, J.A., Franklin, J., Gottschall, L., Hrovatin, L.A., Parker, S., et al. (2001). A new approach to monitoring exercise training. Journal of Strength and Conditioning Research, 15(1), 109-115.

Girard, O., Mendez-Villanueva, A., \& Bishop, D. (2011). Repeated-sprint ability - Part I: Factors contributing to fatigue. Sports Medicine, 41(8), 673-694.

Gouvêa, A.L., Fernandes, I.A., César, E.P., Silva, W.A., \& Gomes, P.S. (2013). The effects of rest intervals on jumping performance: A meta-analysis on post-activation potentiation studies. Journal of Sports Sciences, 31(5), 459-467.

Ha, C., Choi, M., \& Kim, B. (2009). The kinematical analysis of the taekwondo sparring players' bandal chagi in kinematics. International Journal of Applied Sports Science, 21(1), 115-131.

Hopkins, W. (2006). A scale of magnitudes for effect statistics. Retrieved October 7, 2013 from: http://www.sportsci. org/resource/stats/effectmag.html

Kazemi, M., Waalen, J., Morgan, C., \& White, A.R. (2006). A profile of Olympic taekwondo competitors. Journal of Sports Science and Medicine, 5(CSSI), 114-121.

Kilduff, L.P., Bevan, H.R., Kingsley, M.I., Owen, N.J., Bennett, M.A., Bunce, P.J., et al. (2007). Postactivation potentiation in professional rugby players: Optimal recovery. Journal of Strength and Conditioning Research, 21(4), 1134-1138.

Kwok, H.H.M. (2012). Discrepancies in fighting strategies between taekwondo medalists and non-medalists. Journal of Human Sport and Exercise, 7(4), 806-814.

Laurent, C.M., Green, J.M., Bishop, P.A., Sjökvist, J., Schumacker, R.E., Richardson, M.T., et al. (2011). A practical approach to monitoring recovery: Development of a perceived recovery status scale. Journal of Strength and Conditioning Research, 25(3), 620-628.

Mendez-Villanueva, A., Hamer, P., \& Bishop, D. (2008). Fatigue in repeated-sprint exercise is related to muscle power factors and reduced neuromuscular activity. European Journal of Applied Physiology, 103(4), 411-419.

Miarka, B., Del Vecchio, F.B., \& Franchini, E. (2011). Acute effects and postactivation potentiation in the special judo fitness test. Journal of Strength and Conditioning Research, 25(2), 427-431.

Pieter, F., \& Pieter, W. (1995). Speed and force in selected taekwondo techniques. Biology of Sport, 12(4), 257-266.

Sale, D. (2004). Postactivation potentiation: Role in performance. British Journal of Sports Medicine, 38(4), 386-387.

Santos, J.F.S., Valenzuela, T.H., \& Franchini, E. (2015). Can different conditioning activities and rest intervals affect the acute performance of taekwondo turning kick? Journal of Strength and Conditioning Research, 29(6), 1640-1647. 
Tillin, N.A., \& Bishop, D. (2009). Factors modulating post-activation potentiation and its effect on performance of subsequent explosive activities. Sports Medicine, 39(2), 147-166.

Wilson, J.M., Duncan, N.M., Marin, P.J., Brown, L.E., Loenneke, J.P., Wilson, S.M., et al. (2013). Meta-analysis of postactivation potentiation and power: Effects of conditioning activity, volume, gender, rest periods, and training status. Journal of Strength and Conditioning Research, 27(3), 854-859.

Submitted: April 4, 2015

Accepted: January 22, 2016

Correspondence to:

Jonatas Ferreira da Silva Santos

ORCID ID: 0000-0002-3309-4731

Martial Arts and Combat Sports Research Group

Sport Department, School of Physical Education and Sport

University of São Paulo, São Paulo, Brazil

Av. Prof. Mello Morais, 65, Cidade Universitária

CEP: 05508-030 - São Paulo, SP, Brazil

E-mail: jonatas_contato@hotmail.com 